\title{
Collaboration Game Theory Heterogeneous Network Resource Allocation Algorithm
}

\author{
Jianfei Zhou and Na Liu \\ Admission and Employment Office, Chongqing Industry Polytechnic College, \\ School of Information Engineering, Chongqing Industry Polytechnic College \\ fn219@qq.com,na814@qq.com
}

\begin{abstract}
With the continuous improvement of the transmission rate and the quality of wireless communications services, the need for a wireless communication network structure to adjust. The traditional rules of cellular networks no longer meet the high-density, large number of people for high-speed data transmission. Heterogeneous network can solve the problem of network coverage and network-efficient transport, but because of the heterogeneous network dense arrangement of the network, so the introduction of a number of inter-cell interference, especially at the edge of the cell. To solve this problem, we use collaborative tools of game theory, to be distributed among the power cells to determine the coverage of each pico cell. But the need to optimize collaboration between cells, select the number and scope of the coordinated cell. Simulation results show that the proposed algorithm can effectively improve the transmission rate of the system, eliminating the interference between cells.
\end{abstract}

Keywords: LTE-A two-tier network, heterogeneous network, resource allocation, Game theory.

\section{Introduction}

With the widespread adoption of wireless communication technology and the rapid development of intelligent terminals, mainstream business mobile communication system is gradually turning from traditional voice services based multimedia research data show that multimedia services with a strong agglomeration characteristics occurred in the indoor or outdoor hot office area, therefore, the conventional mobile communication system will be difficult to $\mathrm{W}$ meet the growing demand for higher data rates and quality of coverage. To meet these challenges, the Third Generation Partnership Project proposed the concept of heterogeneous networks in advanced Long Term Evolution of standardization. Heterogeneous network is defined as: different network access technologies, different network architectures, different transmission schemes or different coverage access point configuration. According to the above definition, a cellular network capable of future heterogeneous network characteristics, via a macro base station and the small cellular base station, such as micro-cellular base station / femtocell forms femtocell overlapping coverage / isomerization to shorten the cellular network for mobile users and the communication distance between the base station, to solve the traditional network coverage "blind" and "busy area" problem honeycomb, however obtained, heterogeneous cellular networks to provide users than traditional cellular networks higher spectral efficiency for higher data rates It also allows wireless communication environment has become more complex. The Femtocell join the network interference problems become more serious. Heterogeneous networks with the macrocell base station network deployment scenarios, covering an area of $\mathrm{W}$ and transmit power and other aspects quite different. MBS planning and deployment by the operators, the coverage area can be modeled as a regular hexagon. However, the deployment of LBS irregular, uneven 
coverage area. FBS installed by the user, especially in the interior, unpredictable power base station FBS and shutdown time. LBS dispersed or distributed in the form of aggregated MBS coverage area of association "embedded" within the associated area of MBS. HetNet network model is not yet clear conclusion, but W is clearly based on hexagonal grid model can not be applied. Research and analysis Most researchers use PPP to HetNet modeling. With the PPP model of stochastic modeling can be used geometric theory of conclusions and analysis tools. Assuming that all the base stations are independent of position, and each of the base stations have different transmission power and SINR distribution density limits PPP model can be used to obtain closed-form solution HetNet of SINR. In fact, the distribution of base stations in a network deployment is often not completely independent. Based on the analysis results PPP model can be seen as a case of poor SINR (usually no worse than the completely random). In addition, in interference limited situation, increasing the base station does not change HetNet downlink SINR. The industry also proved on Qualcomm verify the probability of the macro cellular network to increase in PBS can increase network capacity without changing SINR distribution [1]. Due to serious interference problems and irregular deployment, resource management HetNet network is more complex than the cellular network. Dense and heterogeneous network is a severe test resource management algorithm design HetNet networks need to be addressed. Meanwhile, HetNet network is difficult to set a unified centralized control also, and higher computational complexity of the centralized algorithm [2]. Using game theory, the theory of self-organization technologies such as distributed resource management algorithms attention of researchers, the algorithm does not require centralized information processing, channel state of each node in the network through the sharing of information between the base station or user information obtained and according to the channel state information to interfere with the independent resource allocation, intelligent avoidance of adjacent nodes generated spectrum efficiency. But this also distributed algorithm to increase in the signaling overhead. In addition, the resource management algorithm based on cognition have also been widespread concern, a base station or a user through cognitive sensing channel state information technology, make full use of idle channel. This method can not only reduce interference, the base station can also be perceived or using a non-authorized band. The traditional homogeneous network users based on the maximum SINR selecting a base station access network can provide users with maximum $\mathrm{W}$ higher data transfer rates. HetNet an MBS transmission power far greater than the LBS, LBS even if the distance is closer to the user received from the maximum SINR may MBS. If the user is still based on the maximum SINR selecting a cell, most users still choose to access MBS. This will cause the MBS still too busy and too LBS idle, resulting in waste of resources LBS. Also in this way will result in some MBS user uplink and downlink asymmetry, the maximum downlink SINR distance LBS that is closer to the user received from the MBS, which is the maximum uplink SINR from LBS, based on the maximum downlink SINR access policy will for LBS serious uplink interference[3]. Therefore, the need to design new cell related manner, the user diverted to LBS, solve the problem of asymmetric uplink and downlink. Load balancing between MBS and LBS, improve resource use efficiency.

\section{Related Work}

\subsection{Game Theory}

Game Theory is regarded as an important branch of applied mathematics and applied science, which has been widely used in various fields of social science. It is a mathematical theory and method for the struggle or competition phenomena in the nature [4].

The strategic form of Game has three main elements: players set, strategies set and the 
Game utility, which can be defined as follows [5]:

- $\mathrm{N}$ is the players set: $\mathrm{N}=\{1, \ldots, \mathrm{N}\}$;

- $\mathrm{S}_{\mathrm{i}}$ is the strategies set of player $\mathrm{i}$;

- $\mathrm{U}_{\mathrm{i}}: \mathrm{S}_{\mathrm{i}} \rightarrow \mathrm{S}$ is the payoff function of player $\mathrm{i}$, in which $\mathrm{S}=\mathrm{S}_{1} \times \ldots \times \mathrm{S}_{\mathrm{i}} \times \ldots \times \mathrm{S}_{\mathrm{N}}$.

Each player, $i$, chooses his own strategy from $S_{i}$, to make the maximum utility with the payoff function, $U_{i}$. The final equilibrium state is the solution of Game, and we will introduce the solutions of the non-cooperative Game and cooperative Game as follows.

(1) Solution of non-cooperative Game: Nash Equilibrium

The solution of non-cooperative Game is called as Nash Equilibrium [6]. Strictly speaking, Nash Equilibrium is a state of non-cooperative Game, in which player, i, cannot improve his utility by changing his strategy if other players do not change their strategies. As for Nash Equilibrium, the strategy combination, $S^{*}=\left(S_{i}^{*}, S_{-i}^{*}\right) \in S$, represents the Nash Equilibrium if and only if the following equation is true:

$$
\mathrm{U}_{\mathrm{i}}\left(\mathrm{S}_{\mathrm{i}}^{*}, \mathrm{~S}_{-\mathrm{i}}^{*}\right) \geq \mathrm{U}_{\mathrm{i}}\left(\mathrm{S}_{\mathrm{i}}, \mathrm{S}_{-\mathrm{i}}^{*}\right), \forall \mathrm{S}_{\mathrm{i}}^{*} \neq \mathrm{S}_{\mathrm{i}}, \forall \mathrm{S}_{\mathrm{i}}^{*}, \mathrm{~S}_{\mathrm{i}}, \mathrm{S}_{-\mathrm{i}}^{*} \in \mathrm{S}, \mathrm{i} \in \mathrm{N} \quad \text { (Equation 1) }
$$

Equation 1 shows that in Nash Equilibrium, each player cannot get higher utility by unilaterally changing the strategy.

(2) Solution of cooperative Game

There are a lot of the concepts for the solution of cooperative Game, and there is no a solution has the similar core status with Nash equilibrium in non-cooperative Game. In the existing solutions of cooperative Game, the better known ones are: core, stable set, Shapley value, bargaining set, Kernel, nucleolus, Nash bargaining solution, and so on.

\subsection{Resource Allocation Algorithms based on Non-cooperative Game}

The representative resource allocation algorithms based on non-cooperative Game in the wireless network are: the interference management algorithm of the femto base station network based on potential Game theory [7], the distributed power control algorithm of the femto base station based on Game theory [8], the resource allocation algorithm of the femto base station based on Stackelberg Game [9].

The interference management algorithm of the femto base station network based on potential Game theory is proposed in literature [7]. It decreases the in-layer interference between the femto base stations and the inter-layer interference between the femto base station and the macro base station by effectively allocating the spectrum RB, and measures the user utility with their average throughput. The literature takes the payoff function as the potential function, and proves the non-cooperative Game to be a potential Game. According to the nature of potential Game, it always converges to Nash equilibrium. The simulation results show that the proposed algorithm has better performance than the random allocation algorithms; however, its advantage is that the down link is not taken into consideration.

The distributed power control algorithm of the femto base station based on Game theory is proposed in literature [8]. It introduces a distributed power control algorithm based on Game theory, which modeling the non-cooperative Game with inter-layer interference and in-layer interference of the downlink. It improves the fairness of the femto base station users and reduces the interference by defining the appropriate utility function. The scene considered in this literature is a central macro base station and a certain amount of femto base stations deployed in the scope of it. The macro base station and femto base stations use the same spectrum, and interference always exist between them due to the distribution randomness of the femto base stations. It shows that the Game in literature [8] is a supermodel, and the algorithm can eventually converges to Nash equilibrium. Each femto base station gets the best transmission power through successive iterations. This algorithm is better than the full power ones in fairness and system capacity; however, it does not consider the interference to the macro base station 
from femto base stations, and the simulation environment is too simple, which cannot support a convincible conclusion.

The resource allocation algorithm of the femto base station based on Stackelberg Game is proposed in literature [9]. It introduces the concept of interference power limitation in the CR network to design the interference control in the two-tier network. In the uplink, macro base station controls the interference from the femto base station users by pricing. It sends the "interference price" to the femto base station through back haul link, and the femto base station sends it to each user. Users can set their transmit power according to the pricing. It means that macro base station controls the transmit power of the femto base station users in the uplink and downlink. At the same time, Stackelberg maximizes the utility of the macrocell and each femto base station user. In the Stackelberg Game, macro base station is the leader, and femto base station users are the followers, and an effective resource allocation strategy is put forward based on pricing. From the perspective of utility maximization of macro base station, disunity pricing is optimized; however, from the perspective of the data transfer rate of the whole femto base station users, unified pricing is optimized. The disadvantage of this literature is that the interference to the femto base station from users is not taken into account. Meanwhile, when the largest interference MBS withstand is not appropriate, the convergence performance cannot be guaranteed.

\subsection{Resource Allocation Algorithms based on Cooperative Game}

The appearance of cooperative Game offers a new solution to a series of problems in the wireless communication. A distributed and autonomous network can apply cooperative Game theory to analyze and research the behavior and interactivity among the network nodes. Literature [10] proposes a distributed spectrum sharing strategy based on cooperative Game. Also, as a branch of cooperative Game, coalitional Game widely applied in the field of wireless communications currently. The representative one is the interference management strategy of recursive core method based on the coalition formation [11].

The distributed spectrum sharing strategy based on cooperative Game is proposed in the scene which requires fair spectrum allocation in multi-hop wireless networks [10]. In high interference environment, the utility function of Game theory is a non-convex function, but with the increase in the number of available channel, the payoff function becomes closer to the convex function, which can achieve the optimal spectrum allocation by strategies. The literature tries to achieve the compromise of fairness and effectiveness by Nash Equilibrium, and puts forwards a distributed algorithm of spectrum sharing to achieve the spectrum allocation strategy close to Nash Equilibrium. This literature firstly analyzes the payoff function and if effective spectrum allocation can be achieved when the channel number increasing. The research does not draw too much attention to the Game process between players, but focuses on achieving the state of Nash Equilibrium. From the simulation results, the cooperative Game is fair and efficient; however, the disadvantage of this literature is that no effective algorithm is put forward for improving system capacity and spectrum efficiency.

In literature [11], the author puts forward a new cooperative Game model to improve the network performance by sharing the the spectrum resources, minimizing the impact rate and maximizing the spectrum reuse rate for femtocell. It models the femtocell spectrum sharing problem based on coalition Game, and proposes a distributed algorithm to form coalition between femtocells. With the proposed algorithm, femtocell can independently decide the cooperation and self-organize the network partition (consisting of unrelated femtocell combination), thus forming a stable partition (depending on the core recursion of Game). In a femtocell coalition, all of the spectrum resources occupied in the femtocell cooperation can schedule their emission mechanism with the distributed way to reduce collisions between each other. The purpose of this algorithm is that 
femtocell can choose coalition which maximize the utility and improve the user performance compared with the non-cooperative Game algorithm in a certain extent. The disadvantage of this paper is that using the coalition Game that can transfer the utility is not reasonable, because the femtocell is separate individuals, and the utility cannot be mutual transferred between each other.

\subsection{Summary}

Non-cooperative Game theory is widely applied in the wireless communication network. However, in non-cooperative Game, users are always selfish, so the utility of the users may not be the maximum even if the Nash equilibrium is achieved. The cooperative Game is to make the utility maximum by cooperation between femto base station users. The cooperative Game algorithms in this section perform better than non-cooperative algorithms. Therefore, our research focuses on the resource allocation algorithm based on cooperative Game.

\section{An Algorithm for Resource Allocation and Interference Management}

The previous section summarizes the application of resource allocation algorithms based on Game theory in the wireless communication network; however, some disadvantages still exist in these algorithms. Aiming at these disadvantages, we put forward a resource allocation and interference management algorithm based on cooperative Game, mainly to reduce the downlink interference of the femto base station from its surrounding users, ensure the QoS and improve the spectral efficiency at the same time.

\subsection{LTE-Advanced Two-tier Network Model}

The two-tier network model is shown in Figure 1, the femto base stations in LTE-A two-tier network are connected into Internet through back haul link, and the downlink applies OFDMA multiple access method. The deployment rate and available rate are represented by $\mathrm{P}_{\mathrm{d}}$ and $\mathrm{P}_{\mathrm{e}}$. The total bandwidth is $\mathrm{B}$, which is divided into $\mathrm{C} R \mathrm{RB}$ (Resource Block). $\mathrm{RB}$ is the minimize spectrum resource unit, and $\mathrm{C}=\{1, \ldots, \mathrm{C}\}$ represents for the RB set.

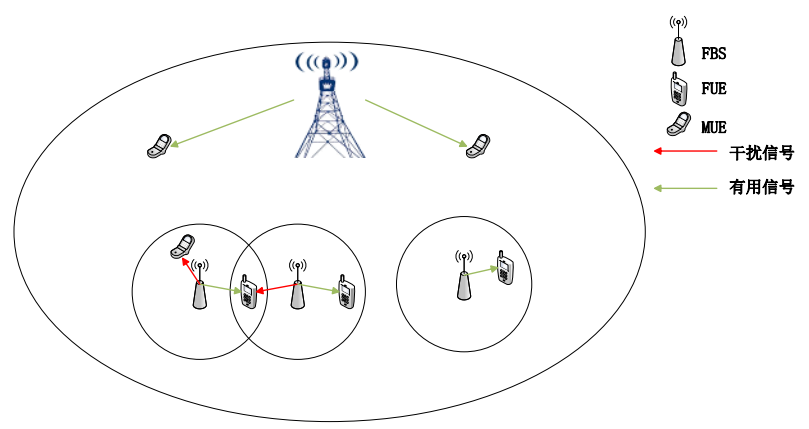

Figure 1. LTE-A Two-tier Network Model

The SINR of femto base station $\mathrm{j}\left(\mathrm{FUE}^{\mathrm{j}}\right)$ in the cth RB can be represented as:

$$
g_{j c}=\frac{p_{j j c} h_{j j c}}{\sum_{i=1, j \leq j}^{N} p_{i j c} h_{i j c+s}}
$$

(Equation 2)

in which $\mathrm{p}_{\mathrm{ijc}}$ represents for the power $\mathrm{FBS}^{\mathrm{i}}$ transmits to $\mathrm{FUE}^{\mathrm{j}}$ in the cth RB, and $h_{i j c}$ represents for the channel gain of FBS $^{i}$ in the cth RB. $\sigma^{2}$ represents for thermal 
noise power. When $\mathrm{i}=\mathrm{j}, \mathrm{FBS}^{\mathrm{i}}$ is the service base station for $\mathrm{FUE}^{\mathrm{j}}$.

The limited throughput of $\mathrm{FUE}^{\mathrm{i}}$ in the cth $\mathrm{RB}$ can be represented as $\mathrm{R}_{\mathrm{ic}}$, and with the Shannon truncation function [9], the throughput of cRB can be represented as:

$$
\mathrm{R}_{\mathrm{ic}}[\mathrm{bps} / \mathrm{Hz}]=\left\{\begin{array}{ccc}
0 & \mathrm{~g}_{\mathrm{ic}}<\mathrm{g}_{\min } \\
\mathrm{aB} \mathrm{BB}_{\mathrm{RB}} \log _{2}\left(1+\mathrm{g}_{\mathrm{ic}}\right) & \mathrm{g}_{\min }<\mathrm{g}_{\mathrm{ic}}<\mathrm{g}_{\max } \\
4.4 \mathrm{~B}_{\mathrm{RB}} & \mathrm{g}_{\mathrm{ic}}>\mathrm{g}_{\max }
\end{array}\right. \text { (Equation 3) }
$$

in which a $=0.6$ represents for the attenuation loss, $g_{\min }=-10 \mathrm{~dB}$ and $g_{\max }=22 \mathrm{~dB}$, $B_{R B}$ represents for the band width of each $R B$.

The limited rate of $F U E^{i}$ is $R_{i}$ :

$$
\mathrm{R}_{\mathrm{i}}=\mathrm{a}_{\mathrm{cîC}} \mathrm{R}_{\mathrm{ic}}
$$

(Equation 4)

Once the transmit power of FBS increases, it will cause co-frequency interference to FUE in the neighbor cell site, and thus there throughput will decrease. In order to improve the performance of femtocell network, the femtocell with severe interference cooperates with the interference sources. The cooperation requires the negotiation process, and the information interaction in it leads to the power consumption, which can be represented as:

$$
P_{i}^{c}=\sum_{j=1, j \neq i}^{M} \beta\left(d_{i, j}\right) P_{i}^{T}
$$

(Equation 5)

in which $d_{i, j}$ represents for the distance between femtocell ${ }^{i}$ and its potential cooperator j. $\beta\left(d_{i, j}\right)$ refers to the distance factor related to $d_{i, j}$, in which the larger $d_{i, j}$ is, the larger $\beta\left(d_{i, j}\right)$ will be. $M$ refers to the times femtocell ${ }^{i}$ requires cooperating. $P_{i}^{T}$ is the transmit power of FBS $^{\mathrm{i}}$, and its maximum and minimum are $\mathrm{P}_{\max }$ and $\mathrm{P}_{\min }$. Lastly, $\mathrm{P}_{\mathrm{i}}^{\mathrm{c}}$ refers to the power consumption in the negotiation process.

The effective power for data transmission of femtocell ${ }^{\mathrm{i}}$ is:

$$
\begin{gathered}
P_{i}=\max \left[0, \min \left(P_{i}^{T}-P_{i}^{c}, P_{i}^{T}\right)\right] \\
P_{i}=\sum_{c=1}^{C_{i}} P_{i i c}
\end{gathered}
$$

We use $\gamma_{\text {th }}$ to represent the minimum SINR for communication of each sub-channel. For FUE ${ }^{\mathrm{i}}$ in sub-channel c, its SINR should meet equation 8 as:

$$
\gamma_{\text {ic }}>\gamma_{\text {th }}
$$

(Equation 8)

Equation 8 replies the minimum SINR for $\mathrm{P}_{\mathrm{i}}$, and $\mathrm{P}_{\mathrm{i}}^{\mathrm{c}}$ should meet:

$$
\begin{gathered}
0 \leq \mathrm{P}_{\mathrm{i}}^{\mathrm{c}} \leq \mathrm{P}_{\mathrm{i}}^{\text {th }} \\
\mathrm{P}_{\mathrm{i}}^{\text {th }}=\mathrm{P}_{\mathrm{i}}^{\mathrm{T}}-\sum_{\mathrm{c}=1}^{\mathrm{C}_{\mathrm{i}}}\left[\left(\sum_{\mathrm{j}=1, \mathrm{j} \neq \mathrm{i}}^{\mathrm{N}} \mathrm{p}_{\mathrm{jic}} \mathrm{h}_{\mathrm{jic}}+\sigma^{2}\right) \gamma_{\mathrm{th}} / \mathrm{h}_{\mathrm{iic}}\right]
\end{gathered}
$$

It is necessary to weigh the power consumption in the negotiation and communication. Once the cooperative coalition is set up, the players in it can negotiate the spectrum resources and transmit power, and schedule their strategies to prevent frequency interference. 


\subsection{Coalitional Game Formulation}

This section introduces the coalitional Game formulation with the framework in cooperative Game [12].

\section{(1) Femtocell coalitional Game}

In order to improve the system capacity of the femtocell network, the appropriate cooperative strategies should be designed. The cooperative model in femtocell can be regarded as the coalitional formation Game, in which femto base station is the player. For each $\mathrm{FBS}^{\mathrm{i}}$ in the coalition $\mathrm{S}$, the utility, $\mathrm{x}_{\mathrm{i}}(\mathrm{S})$, of each player is:

$$
\begin{gathered}
\mathrm{x}_{\mathrm{i}}(\mathrm{S})=\mathrm{R}_{\mathrm{i}}(\mathrm{S})-\alpha \mathrm{P}_{\mathrm{i}}^{\mathrm{T}}(\mathrm{S}) \\
\mathrm{P}_{\mathrm{i}}^{\mathrm{T}}(\mathrm{S})=\mathrm{P}_{\mathrm{i}}(\mathrm{S})+\mathrm{P}_{\mathrm{i}}^{\mathrm{c}}(\mathrm{S}) \\
\mathrm{P}_{\mathrm{i}}(\mathrm{S})=\sum_{\mathrm{c}=1}^{\mathrm{C}_{\mathrm{i}}} \mathrm{p}_{\mathrm{iic}} \\
\mathrm{R}_{\mathrm{i}}(\mathrm{S})=\sum_{\mathrm{c}=1}^{\mathrm{C}_{\mathrm{i}}} \mathrm{B}_{\mathrm{c}} \log _{2}\left[1+\frac{\mathrm{p}_{\mathrm{iic}} \mathrm{h}_{\text {iic }}}{\sum_{\mathrm{j}=1, \mathrm{j} \neq \mathrm{i}}^{\mathrm{N}} \mathrm{p}_{\mathrm{jic}} \mathrm{h}_{\mathrm{jic}}+\sigma^{2}}\right]
\end{gathered}
$$

in which $\mathrm{P}_{\mathrm{i}}^{\mathrm{T}}(\mathrm{S})$ refers to the transmit power of $\mathrm{FBS}^{\mathrm{i}}$, the power consumption in coalition formulation and the power for communication. $\alpha$ is a weigh factor, and $R_{i}(S)$ refers to the throughput of $\mathrm{FBS}^{\mathrm{i}}$ in coalition $\mathrm{S}$.

For coalition $S$, its utility function $v(S)$ refers to the total utility of all the players in $S$ :

$$
v(\mathrm{~S})=\sum_{\mathrm{i} \in \mathrm{S}} \mathrm{X}_{\mathrm{i}}(\mathrm{S})
$$

The coalitional Game with non-transferable payoff [13] is defined as $(\mathcal{N}, \mathrm{V})$, in which $\mathrm{N}$ refers to the players set, and $\mathrm{V}$ refers to the mapping. For each $\mathrm{S} \in \mathcal{N}, v(\mathrm{~S})$ is a closed convex subset of $\mathbb{R}^{S}$, including the utility vector of all players in coalition $S$.

It should be noted that coalitional Game is regarded as non-transferable payoff, so the each player has his own utility, which cannot be transferred to other players in S. So in the coalitional Game with non-transferable payoff, the utility function $v(S)$ is the utility vectors set. To be specifically, element $x_{i}$ in $v(S)$ refers to the utility that the player $i$ gets with some strategy. The utility players in $S$ get are determined by their negotiation result.

Literature [14] defines the concept of coalitional Game in partitions, in which the utility of coalition $S$ is determined by the partition structure, or in another word, by players out of $\mathrm{S}$. We apply $\mathcal{B}$ to represent the coalition structure, also a partition of $\mathcal{N}$, in which $\mathcal{B}=\left\{\mathrm{S}_{1}, \ldots, \mathrm{S}_{1}\right\}, \quad \forall \mathrm{i} \neq \mathrm{j}, \mathrm{S}_{\mathrm{i}} \cap \mathrm{S}_{\mathrm{j}}=\emptyset$, and $\mathrm{U}_{\mathrm{i}=1}^{\mathrm{l}} \mathrm{S}_{\mathrm{i}}=\mathcal{N}$. The utility of $\mathrm{S}$ (included in $\mathcal{B})$ is $v(\mathrm{~S}, \mathcal{B})$.

The utility in equation 15 is a specific value, and the femtocells in the same coalition cannot transfer their utility. Similarly, we take the coalitional Game in partitions as coalitional Game with non-transferable payoff. In this way, for a coalition $S$ in $\mathcal{B}$, the utility set, $v(S, \mathcal{B})$, is single element set, which can be expressed as:

$$
\mathrm{V}(\mathrm{S}, \mathcal{B})=\left\{\begin{array}{ll}
v(\mathrm{~S}) \mid \mathrm{x}_{\mathrm{i}}(\mathrm{S}) \in v(\mathrm{~S}), \forall \mathrm{i} \in \mathrm{S}, \mathrm{S} \subseteq \mathcal{N}, \mathrm{S} \in \mathcal{B} & \mathrm{P}_{\mathrm{i}}^{\mathrm{c}}(\mathrm{S}>0) \\
0 & \text { otherwise }
\end{array} \quad\right. \text { (Equation 16) }
$$

With the above definition, we get the coalitional Game model in partitions with non-transferable payoff of femtocell. In the following, we will propose a distributed algorithm to find the optimized partition to make the coalition utility best.

\section{(2) The problem formulation}

Elements in the coalition set $\mathcal{B}=\left\{\mathrm{S}_{1}, \ldots, \mathrm{S}_{\mathrm{l}}\right\}$ are mutually disjoint, namely that different coalitions can reuse the spectrum. The coalition structure is determined by the 
femtocell network deployment. With some external reasons, such as inter-layer interference, femtocell may get coalitions in any form. The utility function of Femtocell ${ }^{\mathrm{i}}$ refers to its throughput, with the power consumption in the negotiation process considered.

The utility maximization problem of femtocell can be expressed as:

$$
\begin{array}{cc}
\max \sum_{\mathrm{S}_{\mathrm{i}} \in \mathcal{B}} \sum_{\mathrm{j} \in \mathrm{S}_{\mathrm{i}}} \mathrm{x}_{\mathrm{j}}\left(\mathrm{S}_{\mathrm{j}}\right) & \text { (Equation 17) } \\
\text { s.t. } \mathrm{P}_{\min } \leq \mathrm{P}_{\mathrm{j}}\left(\mathrm{S}_{\mathrm{i}}\right) \leq \mathrm{P}_{\text {max }}, 0 \leq \mathrm{P}_{\mathrm{j}}^{\mathrm{c}} \leq \mathrm{P}_{\mathrm{j}}^{\text {th }} & \text { (Equation 18) }
\end{array}
$$

In order to solve the utility maximization problem, femtoell ${ }^{\mathrm{i}}$ sends the cooperation request to the interference sources, namely the neighbor femtocells, to form the coalition. In this coalition, femtocells can negotiate to use the spectrum resources and schedule the transmit power, thus to reduce the interference between them. In particular, as for the limited spectrum resources and the increase of power consumption in information communication, the coalition size is always not too large, and the players are always not too many.

\subsection{The Distributed Coalition Formulation Algorithm}

Firstly, the definition of preference relation should be introduced [15]: preference relation, > , is to compare two sets: $\mathcal{R}=\left\{\mathrm{R}_{1}, \ldots, \mathrm{R}_{1}\right\}, \mathcal{S}=\left\{\mathrm{S}_{1}, \ldots, \mathrm{S}_{1}\right\} . \mathcal{R}$ and $\mathcal{S}$ refers to two different partition forms, and $\mathcal{R}^{>} \mathcal{S}$ means that $\mathcal{R}$ prefers than $\mathcal{S}$.

Pareto Sequence will also applied in this paper, which is defined as: given two utility sets $\mathrm{x}$ and y refers to the utility of $\mathcal{R}$ and $\mathcal{S}$, when and only when $\mathrm{x}>y, \mathcal{R}>\mathcal{S}$. Pareto Sequence is an individual value sequence. If players choose $\mathcal{R}$ rather than $\mathcal{S}$, it means $\mathcal{R}$ provides better utility of at least one player without nobody's utility getting worse. In the coalitional Game with non-transferable payoff, we need to find a structure with Pareto optimal distribution.

Moreover, the solution of "merge and split" should also be introduced [16]:

- Merge Rule: once the utility after merging is better, any coalition in the coalitions set $\left\{\mathrm{S}_{1}, \ldots, \mathrm{S}_{1}\right\}$ starts to merge. It also means:

$$
\text { when }\left\{U_{j=1}^{l} S_{j}\right\}>\left\{S_{1}, \ldots, S_{l}\right\},\left\{S_{1}, \ldots, S_{l}\right\} \rightarrow\left\{U_{j=1}^{l} S_{j}\right\}
$$

- Split Rule: once the utility after splitting is better, any coalition in the coalitions set $\left\{\mathrm{S}_{1}, \ldots, \mathrm{S}_{\mathrm{l}}\right\}$ starts to split. It also means:

$$
\text { when }\left\{U_{j=1}^{1} S_{j}\right\} \rightarrow\left\{S_{1}, \ldots, S_{l}\right\},\left\{S_{1}, \ldots, S_{l}\right\}>\left\{U_{j=1}^{1} S_{j}\right\}
$$

According to the definition of Pareto Sequence, when and only when at least the utility of at least one femtocell can be improved without impairing the utility of other femtocells, the coalition will merge and split. In coalitional Game, Femtocells form the coalition by interaction and finally converge to a steady partition state, in which all the femtocells get their optimized utility and no one wants to break it. The steps of this algorithm are as follows:

\section{(1) The initialization status:}

- At the beginning, the partition form is $\mathcal{S}=\mathcal{N}=\{1, \ldots, \mathrm{N}\}$, namely all the femtocells are in the non-cooperative mode.

- With some known techniques [17], each femtocell takes the interference sources as the potential cooperators. 


\section{(2) The iteration process:}

- Each femtocell sends cooperation request to potential cooperators, and negotiate in pairs.

- Each femtocell determines the coalitions it may join in.

- Compute the utility in different coalitions of the femtocell with equation 16.

- According to the merge and split strategy, the femtocells form coalitions and join in the coalition providing them the best utility.

- The generated coalitions will be included in a steady partition.

- Iteration the above steps until all femtocells are converged to a steady partition.

\subsection{The Coalition Partition Stability}

Once the coalition formation algorithm in femtocell network is established, the coalition partition stability should be discussed. So the definition of "defection function" is introduced, to evaluate the partition stability of femtocell [18]. It is defined as follows: defection function, $\mathbb{D}$, is a function relevant to each partition, $\mathcal{S}=\left\{\mathrm{S}_{1}, \ldots, \mathrm{S}_{1}\right\}$ (in which $S_{i}$ refers to a coalition). When all the femtocells have no will to leave the partition $\mathcal{S}$, it is called as $\mathbb{D}$-stable.

There are two kinds of defection functions [18], $\mathbb{D}_{\mathrm{hp}}$ and $\mathbb{D}_{\mathrm{c}}$, in which $\mathbb{D}_{\mathrm{hp}}$ is an equilibrium-like stability, and $\mathbb{D}_{\mathrm{c}}$ is more stable than $\mathbb{D}_{\mathrm{hp}}$. When $\mathbb{D}_{\mathrm{c}}$-stable conditions are meet, it can be proved that our algorithm based on merge and split strategies will converge to the optimal strict $\mathbb{D}_{c}$-stable state. However, the $\mathbb{D}_{c}$ stability is determined by the deployment of femtocells in the physical network, and $\mathbb{D}_{c}$-stable state does not always exist. If there's no $\mathbb{D}_{\mathrm{c}}$-stable state our algorithm will converge to the suboptimal $\mathbb{D}_{\text {hp-stable state. }}$

\section{Algorithm Simulation and Experimental Evaluation}

The proposed algorithm will be simulated in this section, and its performance will be evaluated by comparing with the non-cooperative algorithm.

\subsection{Simulation Parameters}

3GPP urban deployment scene is applied in the simulation in this paper, which owns 7 macrocells in hexagon with 3sectors. The femtocells are deployed with Dual Stripe model, and each Stripe is $2 \times 10,6$ floors, and the size of each room is $10 \times 10 \mathrm{~m}$. The femtocell is deployed in each room, and each room owns at most one femtocell. The simulation parameters of the two-tier network are shown in Table 1.

Table 1. Two-Tier Network Parameters

\begin{tabular}{|c|c|c|c|}
\hline Parameter & Value & Parameter & Value \\
\hline Bandwidth & $10 \mathrm{MHz}$ & Thermal Noise Density & $174 \mathrm{dBm} / \mathrm{Hz}$ \\
\hline Carrier Frequency & $2 \mathrm{GHz}$ & MUE Indoor Probability & $0 \sim 100 \%$ \\
\hline Macrocell Radius & $500 \mathrm{~m}$ & FBS Activity Rate & $100 \%$ \\
\hline MBS Transmit Power & $46 \mathrm{dBm}$ & FBS Deployment Rate & $10 \% \sim 100 \%$ \\
\hline FBS Transmit Power & $20 \mathrm{dBm}$ & Penetration Loss & $20 \mathrm{~dB} / 5 \mathrm{~dB}$ \\
(Maximum/Minimum) & $/ 0 \mathrm{dBm}$ & (Outside/Inside) & \\
\hline Shadow Standard & $8 \mathrm{~dB}$ & UE number & $\begin{array}{c}1 \mathrm{FUE} / \mathrm{FBS}, \\
10 \mathrm{MUE} / \mathrm{Sector}\end{array}$ \\
\hline Deviation (FBS/MBS) & $/ 4 \mathrm{~dB}$ & & Full Buffer \\
\hline Antenna & $14 \mathrm{dBi}$ & Communication Model & 50 \\
\cline { 2 - 4 } Gain(FBS/MBS) & $15 \mathrm{dBi}$ & Available RB Number & \\
& & &
\end{tabular}


It should be noted that the access method of femtocell is closed, and MUE are always near to the macrocells. The transmit power homogeneously distributed in RB.

\subsection{Simulation Results and Analysis}

Firstly, we draw the femtocell network cooperation figure as Figure 2, in which the blue points refer to the femto base stations, the pink points refer to the femtocell users, and lines between femto base stations reply a cooperation between them.

Figure 3 shows the relationship between the coalition numbers and femtocell numbers. With the increase of femtocell numbers, the interference between femtocells become more sever, thus leading to the increase in the cooperation.
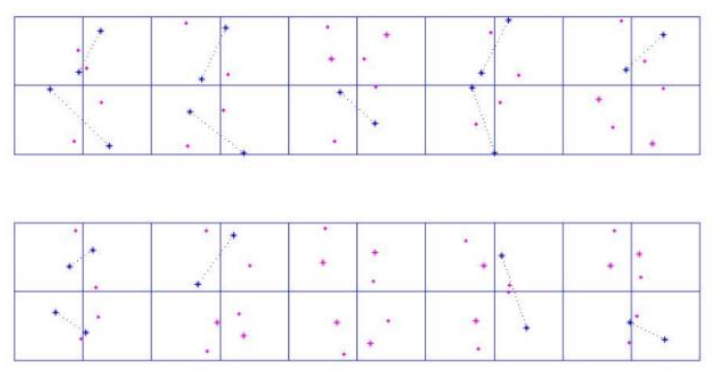

Figure 2. Femtocell Network Cooperation

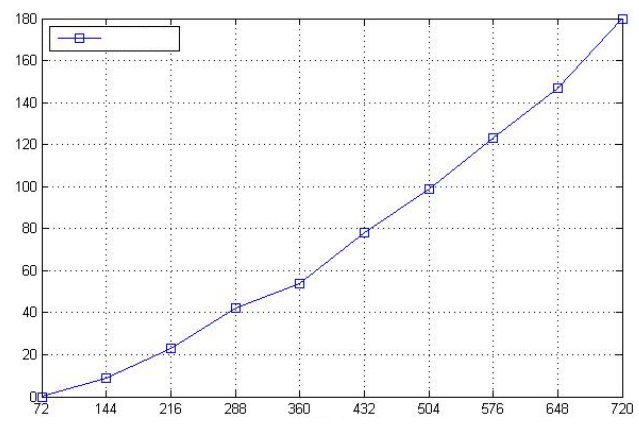

Figure 3. Coalition Numbers and Femtocell Numbers

\section{(1) Cutting off rate of femtocell users}

Figure 4 shows that the cutting off rate increases with the increase of femto base stations. The red line refers the cutting off rate curve in non-cooperative Game algorithm, which climbs steeply with the increase of femto base stations. The blue line refers to the cutting off rate curve in the algorithm proposed in this paper. With the increase of femto base stations, it shows smaller amplitude. It is because that the femtocell users form coalitions with the interference sources with the cooperative Game algorithm, thus reducing the interference.

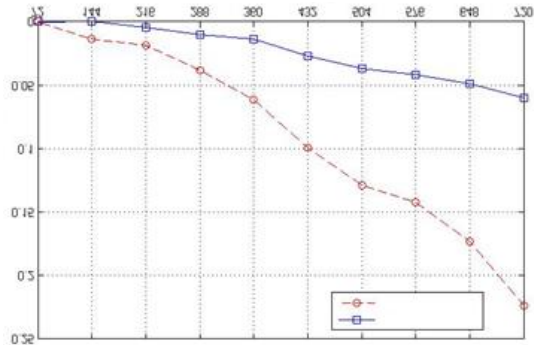

Figure 4. Cutting off Rate of Femtocell Users

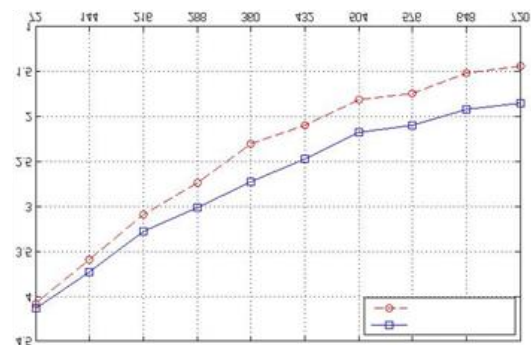

Figure 5. Spectrum Effectiveness of Femtocell Users

\section{(2) Spectrum effectiveness of femtocell users}

Figure 5 shows that our algorithm improves the spectrum effectiveness by $13 \%$ compared with the non-cooperative algorithm, especially deploying more femto base stations. It is because that each femtocell in our algorithm chooses to cooperate and forms coalitions that make its utility maximum. In the coalitions, the players negotiate to use the spectrum resources and transmit power to reduce the inter-layer interference, thus improving the spectrum effectiveness. However, in the non-cooperative algorithm, the 
femto base stations occupy the spectrum resources "selfishly" to improve its own transmit power, meanwhile causing more severe interference to its neighbor cells, and lastly lead to a vicious cycle. It makes the spectrum effectiveness of femtocell users lower than cooperative algorithm with more femto base stations deployed.

\section{Conclusions}

This paper presents a cooperative game theory based on the interference management and resource allocation scheme, the method by player consultation theory game theory, proposed power allocation scheme, through effective collaboration and cell delineation program, consistent with the effective co-channel interference, thereby improving the throughput of the system, while improving the quality of cell edge users of the service, the simulation results show the effectiveness of the method.

\section{References}

[1] S. Parkvall, E. Dahlman and A. Furuskar, "LTE-advanced-evolving LTE towards IMT-advanced", Vehicular Technology Conference, 2008, VTC 2008-Fall. IEEE $68^{\text {th }}, \mathbf{( 2 0 0 8 )}$, pp. 1-5.

[2] A. Damnjanovic, J. Montojo and Y. Wei, "A survey on 3GPP heterogeneous networks", Wireless Communications IEEE, vol. 18, no. 3, (2011), pp. 10-21.

[3] J. Kim, H. Kim and K. Cho, "SON and Femtocell Technology for LTE-Advanced System", International Conference on Wireless \& Mobile Communications, (2010), pp. 286-290.

[4] D. Fudenberg and J. Tirole, "Game Theory", MIT Press, (1993).

[5] G. Owen, "Game theory", Encyclopedia of Applied Ethics, (1995), pp. 391-398.

[6] J. F. Nash, "Equilibrium points in n-person Games", Proceedings of the national academy of sciences, vol. 36, no. 1, (1950), pp. 48-49.

[7] I. W. Mustika, K. Yamamoto and H. Murata, "Potential Game approach for self-organized interference management in closed access femtocell networks", Vehicular Technology Conference (VTC Spring), 2011 IEEE 73rd. IEEE, (2011), pp. 1-5.

[8] E. J. Hong, S. Y. Yun and D. H. Cho, "Decentralized power control scheme in femtocell networks: A Game theoretic approach", Personal, Indoor and Mobile Radio Communications, 2009 IEEE 20th International Symposium on IEEE, (2009), pp. 415-419.

[9] X. Kang, R. Zhang and M. Motani, "Price-based resource allocation for spectrum-sharing femtocell networks: A stackelberg Game approach", Selected Areas in Communications, IEEE Journal, vol. 30, no. 3, (2012), pp. 538-549.

[10] J. E. Suris, L. A. DaSilva and Z. Han, "Cooperative Game theory for distributed spectrum sharing", Communications, 2007, ICC'07, IEEE International Conference on IEEE, (2007), pp. 5282-5287.

[11] F. Pantisano, M. Bennis and W. Saad, "Coalition formation Games for femtocell interference management: A recursive core approach", Wireless Communications and Networking Conference (WCNC), 2011 IEEE. IEEE, (2011), pp. 1161-1166.

[12] W. Saad, Z. Han and M. Debbah, "Coalitional Game theory for communication networks", Signal Processing Magazine, IEEE, vol. 26, no. 5, (2009), pp. 77-97.

[13] R. B. Myerson, "Game theory: analysis of conflict", Harvard university press, (2013).

[14] R. M. Thrall and W. F. Lucas, "N-person Games in partition function", Naval Research Logistics Quarterly, vol. 10, no. 1, (1963), pp. 281-298.

[15] D. Niyato and W. Saad, "Game Theory in Wireless and Communication Networks: Theory, Models, and Applications", Cambridge University Press, (2011).

[16] K. R. Apt and A. Witzel, "A generic approach to coalition formation", International Game Theory Review, vol. 11, no. 03, (2009), pp. 347-367.

[17] D. L. Pérez, A. Valcarce, G. De La Roche, "OFDMA femtocells: A roadmap on interference avoidance", Communications Magazine, IEEE, vol. 47, no. 9, (2009), pp. 41-48.

[18] K. R. Apt and T. Radzik, "Stable partitions in coalitional Games", arXiv preprint cs/0605132, (2006). 


\section{Authors}

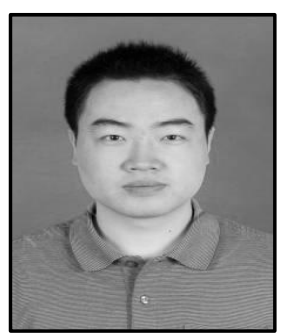

Jianfei Zhou, he received the Bachelor degree in Engineering in College of computer and Information Science from Southwestern Normal University, and the Master's degree of Engineering in Computer Technology field From College of Computer Science of Chongqing University, China in 2004 and 2012 respectively. He is currently researching on Computer network, Information security, Graphics and Image Processing.

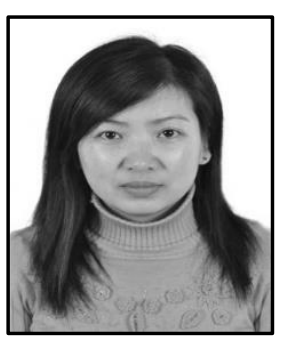

Na Liu, she received the Bachelor degree in Engineering in College of computer and Information Science from Southwestern Normal University, and the Master's degree of Engineering in Computer Technology field From College of Computer Science of Chongqing University, China in 2004 and 2013 respectively. She is currently researching on Database technology, Graphics and Image Processing. 